\title{
Gender Differences and Outcomes in Melanoma Patients
}

\author{
Francesca Morgese - Caterina Sampaolesi · Mariangela Torniai · \\ Alessandro Conti • Nicoletta Ranallo • Alfredo Giacchetti • \\ Stefano Serresi · Azzurra Onofri · Michela Burattini - Giuseppe Ricotti • \\ Rossana Berardi
}

Received: September 25, 2019 / Published online: February 4, 2020

(C) The Author(s) 2020

\section{ABSTRACT}

Introduction: Melanoma is one of the most common cancers in younger people. The incidence of cutaneous melanoma is increasing in patients of both sexes, with female patients generally living longer than their male counterparts. The aim of this retrospective study was to evaluate and confirm the sex-based difference in survival of melanoma patients and the relationship of this difference with pathological features.

Enhanced Digital Features To view enhanced digital features for this article go to https://doi.org/10.6084/ m9.figshare.11568663.

F. Morgese - C. Sampaolesi · M. Torniai · N. Ranallo - A. Onofri · M. Burattini · R. Berardi $(\bowtie)$ Clinica Oncologica, Università Politecnica delle Marche-Azienda Ospedaliero-Universitaria Umberto I, Via Conca, Ancona, AN, Italy e-mail: r.berardi@univpm.it

\section{A. Conti}

Department of Clinical and Specialist Sciences, Urology, Università Politecnica delle Marche-Azienda Ospedaliero-Universitaria Umberto I, Via Conca, Ancona, AN, Italy

A. Giacchetti · S. Serresi · G. Ricotti L'Unità Operativa di Dermatologia, INRCA/IRCCS, Via Montagnola, Ancona, AN, Italy
Methods: A total of 1023 patients who had been treated at the Department of Medical Oncology, Università Politecnica Marche (Ancona, Italy) and the INRCA-IRCCS Department of Dermatology (Ancona, Italy) between 1987 and 2014 were enrolled in the study.

Results: In terms of stage of disease at onset, there was a significant difference in disease-free survival (DFS) and overall survival (OS) in favor of female patients in disease stage I $(P=0.001$ and $P=0.01$, respectively) and II ( $P=0.02$ and $P=0.009$, respectively). Female patients also showed a significant improvement in 12-year DFS and 12-year OS adjusted for pathological features (Breslow thickness, ulceration, "absent" tumor-infiltrating lymphocyte (TIL) melanomas, "non-brisk" TIL pattern). Globally, female patients had an advantage over with male patients in both DFS and OS $(P<0.001)$.

Conclusions: Our results show that women have a survival benefit over with men after adjustment for many variables that can reduce mortality risk in female melanoma patients. In a future investigation we wish to examine possible biological sex differences in tumor-host interactions.

Keywords: Clinical-pathological factors; Gender identity; Melanoma patients 


\section{Key Summary Points}

The incidence of melanoma is increasing dramatically in both females and males, but male melanoma patients have been found to have shorter survival than female melanoma patients.

The aim of this retrospective study, performed on a population of 1023 patients with cutaneous melanoma, was to evaluate the difference in survival between male and female melanoma patients, as well as the relationship of this survival difference with the pathological features of the neoplasm.

The results demonstrate that female melanoma patients do have an advantage over their male counterparts in terms of both disease-free survival and overall survival.

\section{INTRODUCTION}

Cutaneous melanoma is the 19th most common cancer diagnosed in men and women, respectively. In 2018, nearly 300,000 new cases were identified [1]. The annual age-standardized rate of melanoma incidence is reported to be 19.7 per 100,000 cases $[2,3]$. The incidence of melanoma worldwide has increased over the last decades, augmenting rapidly by $4-6 \%$, most commonly in fair-skinned subjects [4-6]. The median age at diagnosis of cutaneous melanoma is 60 years [7]. The incidence rates of melanoma are higher in women than in men younger than 50 years of age; however, the rates in men are twofold and threefold higher at the ages of 65 and 80 years, respectively [5].

In the USA, melanoma accounts for $5 \%$ of the estimated new cases of malignances in men and $4 \%$ in women. It has been estimated that in 2019 there would be about 192,310 diagnoses of melanoma: 95,830 T0 cases [according to the TNM staging of the eighth edition of the 2009
American Joint Committee on Cancer (AJCC) melanoma staging system] and 96,480 invasive cases. Of the estimated number of invasive melanoma cases, about 57,220 are estimated to present in men and 39,260 in women $[5,8]$. The incidence of melanomas continues to increase dramatically and more rapidly in men than in women; in women, melanoma is the secondmost common tumor after lung cancer. In particular, in the USA, an overall incidence rate of $33 \%(+3.5 \% /$ year $)$ for men and 23\% $(+3.6 \% /$ year) for women from 2002 to 2006 was observed [5].

In the European Union, the incidence of melanoma is about nine new cases per 100,000/ year. This incidence tends to increase with latitude, with higher prevalence in populations with poor pigmentation (12-17 per 100,000 cases/year) compared to the populations of Mediterranean countries (3-5 per 100,000 cases/ year). The mortality rate is 2.3 per 100,000 cases/year [9].

In Italy, cutaneous melanoma represents the second most common cancer diagnosis both in males and in females between 0 and 39 years of age. More specifically, in northern regions of Italy, patients with melanoma represent the fifth most frequent cancer patient group; in comparison, melanoma patients rank the tenth most frequent cancer patient group in southern regions of Italy [10, 11]. In 2018, about 13,700 new cases of cutaneous melanoma were estimated, 7200 among males and 6500 among females (4\% of all cancers in both sexes) [12].

The annual incidence of cutaneous melanoma is 14.3 per 100,000 for men and 13.6 per 100,000 for women. Melanoma represents 9\% of juvenile cancers in males (second most frequent neoplasm) and 7\% in females (third most frequent neoplasm). The trend in the incidence of cutaneous melanoma appears to be increasing at a statistically significant rate, both in men $(+3.4 \%$ per year) and in women $(+2.0 \%$ per year) $[12,13]$. The incidence of this neoplasm has doubled in 10 years, while the mortality has remained substantially stable. In Italy, deaths due to melanoma disease accounts for $1 \%$ of all cancer deaths $(1.1 \%$ in men and $0.9 \%$ in women). In recent decades the trend in males has shown a moderate rise $(+2.3 \%$ /year $)$, while 
a substantial stability has been identified in females. In 2014, in Italy there were 2018 deaths from cutaneous melanoma, about $1 \%$ of all deaths due to cancer in both sexes [14].

Additionally, it has been estimated that approximately two-thirds of the patients with melanoma were still alive $\geq 5$ years after diagnosis. Melanoma patients of both sexes and different ages had a time to cure of $4-12$ years [15-17].

The increase in melanoma incidence appears to be linked to an "overdiagnosis" of in situ or thin melanomas associated with a relatively good prognosis without a reduction of incidence of thick forms with worse prognosis $[18,19]$.

Malignant melanoma usually presents a favorable prognosis: during the last two decades, the 5-year survival probability at 1 and 5 years after the diagnosis has increased to 87 and $96 \%$, respectively, in males and to 91 and to $96 \%$, respectively, in females [20]. These data show that women with melanoma generally have a significantly longer survival time than male melanoma patients [21].

The aim of this study was to evaluate and confirm how gender differences can influence disease-free survival (DFS) and overall survival (OS), taking into account specific clinical-pathological features.

\section{METHODS}

\section{Patients' Selection}

This was a retrospective study in which the clinical history and follow-up of patients with malignant melanoma treated at the Department of Medical Oncology, Università Politecnica Marche (Ancona, Italy) and at the INRCAIRCCS Department of Dermatology (Ancona, Italy) between 1987 and 2014 were reviewed. Eligibility criteria included histological diagnosis of malignant melanoma.

Data were retrospectively collected from patients' medical records and included both patient characteristics and clinical-pathological features, such as age, sex, stage of disease classified according to TNM staging as described in the 2009 American Joint Committee on Cancer melanoma staging system [22], site of tumor, histological type, Breslow thickness, histological ulceration and tumor-infiltrating lymphocytes.

According to Italian law (resolution March 1, 2012, Gazzetta Ufficiale n.72 of March 26, 2012), ethics approval and informed consent were not required for the present study owing to its retrospective nature, the use of anonymous data and the fact that it was not associated with any change in patients' management. All patients gave their written consent to all the diagnostic and therapeutic procedures.

\section{Technical Information}

The aim of our study was to assess the impact of gender on OS and DFS. The primary endpoint was to evaluate gender differences in the whole population, following which subgroup analyses were conducted in which patients were stratified according to various other clinical parameters usually using predictive models for melanoma.

The primary outcomes of this study were OS and DFS from diagnosis to event (death or recurrence of disease) or censoring. The secondary aim was to investigate the clinical-pathological features of patients with melanoma. Data were retrospectively collected from medical chart reviews and electronic records.

\section{Statistical Analysis}

Patients' characteristics were stratified according to gender, and the homogeneity of the distribution between the two sexes was assessed by means of the Pearson chi-squared test, applying Yates correction, where appropriate, while pairs of continuous variables were compared with Student's $t$ test. Survival was estimated by the Kaplan-Meier product-limit method, and the Mantel-Haenszel log-rank test was used to compare survival among groups. Median survival and the respective 95\% confidence interval (CI) were reported, when reached. The univariate hazard ratio (HR) with 95\% CIs were 
also estimated and reported for comparison between the two groups, after testing for proportionality of the hazard in time with visual inspection of Schoenfeld's residuals plot for each variable.

The primary endpoints OS and DFS were evaluated from histological diagnosis of melanoma to event or censoring. The selected grouping variables were clinical stage, Breslow thickness, presence of histological ulceration, tumor-infiltrating lymphocytes (TILs) and known established predictors in the general population cited in the literature and used in clinical practice [22-24]. Both continuous variable age and clinical stage were dichotomized for the purposes of this investigation according to previous reports on the validity of the distinction in these groups [25].

Significance was set at the 0.05 level for all analyses, and all $P$ values were two-sided. Statistical analysis was conducted with the "R" statistical software version 3.0.1 (R Foundation for Statistical Computing, Vienna, Austria. http://www.R-project.org/).

\section{RESULTS}

Data were collected on 1023 cases of patients treated for melanoma during the study period. Of these patients, 487 were men. The median age at diagnosis was 54 (range 13-96) years. The patients' characteristics are given in detail in Table 1, and the most important of these according to sex are shown in Table 2, together with the significance level for each group.

A statistically significant higher percentage of men than women presented with histological ulceration (20 vs. $13 \% ; P=0.003$ ), while no significant differences were found in the distribution of TILs between the two sexes. There was also a statistical difference in DFS according to gender, with men having a significantly shorter median DFS (mDFS) than women (22 vs. 104 months; $P<0.001)$ and a significantly shorter median OS (mOS) (20.7 vs. 104 months, respectively; $P<0.001)$. In the subgroup analysis on the effect of age (patients aged $<55$ years vs. those aged $\geq 55$ years), cumulative survival expectancy in both subgroups was higher in female patients, even though mDFS and mOS not reached for most groups of patients due to the low incidence of the events.

The subgroup analysis according to the stage of the disease at the time of diagnosis revealed that there was a statistically significant difference in DFS and OS in favor of females in TMN stages I (median not reached; HR, $P=0.001$ ) and II (mDFS 4 [males] vs. 12 months [females], $P=0.02 ; \operatorname{mos} 7$ [males] vs. 16 months [females], $P=0.009$ ). In the subgroup analysis of patients with TMN stage III and IV melanoma, no significant difference was observed.

In the subgroup analysis according to pathological TNM stage, global DFS and OS were found to be significantly longer in women when patients were stratified according to Breslow thickness (pT1 and pT2); mDFS and mOS were not reached $(P<0.001)$. No statistical difference was established in DFS and in OS for stages pT3 and pT4 shared by gender ( $P=0.19$ and $P=0.09$, respectively), but the limited sample size in this group did not allow any adequate statistical power on which to draw a reliable conclusion.

In the analysis of the subset of patients presenting histological ulceration, we identified a statistically significant advantage for females over males in terms of both mDFS (3.1 vs. 8.8 months; $P=0.01)$ and $\operatorname{mOS}(3.53$ vs. 11.6 months; $P=0.005)$. Similar results were noted in both DFS and OS in patients without histological ulceration, with females showing longer survival $(P<0.001)$; despite the better prognosis for females in this group of patients, however, median survival values were not reached despite the long median follow-up (12.8 months, range 0.079-113 months).

In comparison to males affected by melanoma with "absent" TILs, female patients with the same pathological characteristics showed a better DFS (mDFS median not reached vs. 19.3 months; $P<0.001$ ) and OS (mOS median not reached vs. 20.7 months; $P<0.001)$. Similarly, in comparison to male patients affected by melanoma with "non-brisk" TILs, female patients with the same pathological characteristics also had a better DFS (mDFS 28.6 vs. 21.6 months; $P=0.003$ ) and OS without reaching median values (even though a significant 
Table 1 Clinical-pathological characteristics of all melanoma patients enrolled in the study

Clinical-pathological features of $1023 \quad N$
patients treated for melanoma

Gender

$\begin{array}{ll}\text { Male } & 487(47.6 \%) \\ \text { Female } & 536(52.4 \%) \\ \text { Age (years) } & \\ \text { Median [range] } & 54 \text { [13-96] }\end{array}$

TNM stage (2009 AJCC melanoma staging system)

\begin{tabular}{ll} 
Stage 0 & $181(17.7 \%)$ \\
Stage I & $549(53.7 \%)$ \\
Stage II & $183(18.0 \%)$ \\
Stage III & $40(4.0 \%)$ \\
Stage IV & $23(2.2 \%)$ \\
Unknown/not applicable & $45(4.6 \%)$ \\
Pathological TNM stage (pT) & \\
pTis & $179(17.5 \%)$ \\
pT1 & $429(41.9 \%)$ \\
pT2 & $166(16.2 \%)$ \\
pT3 & $113(11.0 \%)$ \\
pT4 & $82(8.0 \%)$ \\
Unknown/not applicable & $54(5.3 \%)$ \\
Histological ulceration & \\
Present & $132(12.9 \%)$ \\
Absent & $675(66.0 \%)$ \\
Unknown/not applicable & $216(21.1 \%)$ \\
Tumor-infiltrating lymphocytes & \\
Absent & $645(63.0 \%)$ \\
Non-brisk & $257(25.1 \%)$ \\
Brisk & $74(7.2 \%)$ \\
Unknown/not applicable & $47(4.6 \%)$ \\
Disease-free survival (months) & \\
Median [range] & \\
\hline
\end{tabular}

Table 1 continued

Clinical-pathological features of $1023 \quad N$ patients treated for melanoma

Overall survival

\begin{tabular}{l} 
Median \\
\hline Values in table are presented as the number (of patients) \\
with either the range (in square brackets) or the percentage \\
(in parenthesis) \\
AJCC 2009 American Joint Committee on Cancer, N.R. \\
not reached
\end{tabular}

difference was revealed in the log-rank comparison of survival curves, $P=0.009$ ) in comparison to males with the same pathological characteristics. No statistical difference in DFS and OS was found in patients with "brisk" TILs melanomas $(P=0.75$ and $P=0.72$, respectively). A Kaplan-Meier comparison of this analysis is shown in Figs. 1 and 2.

Only $1.8 \%$ of patients underwent basic life support, and only $4.4 \%$ of patients received adjuvant treatment with interferon. Data on systemic therapies were not included in the analysis due to heterogeneity and small sample sizes. In particular, only $2.2 \%$ of patients underwent systemic therapies (especially chemotherapy). Therefore, our results must be examined without any consideration given for the various therapies undergone by the patients.

\section{DISCUSSION}

The most intriguing result of this hospital-based retrospective study was the highly consistent and independent prognostic benefit in terms of melanoma outcome of being female and not male. This advantage was found to be similar across the different endpoints (DFS and OS). Also, in terms of the subgroups analysis, DFS and OS were found to be significantly better in female patients than in male patients, in particular in those patients presenting $\mathrm{T} 1$ and $\mathrm{T} 2$ melanomas, primary cutaneous melanomas (both with ulceration and without), and in 
melanomas with "absent" TILs and with "nonbrisk" TILs.

These gender differences in patients with cutaneous melanoma have not been completely elucidated even though they have been reported both in terms of incidence and of outcome in the most recent surveys. In 2011 in the USA, there were an estimated 70,230 cases of invasive cutaneous melanoma, of which 43\% $(30,220$ cases) occurred in women; however, of the 8790 melanoma-related deaths, only 35\% (3040 deaths) occurred in women [26].

Gender differences in cutaneous melanoma have been the focus of a number of studies in the last few years, with the results indicating that the better survival in women compared to men is independent of the main histological and clinical prognostic factors [27-29]. In a retrospective study involving 10,538 melanoma patients, de Vries et al. showed that men had a relative excess risk of dying of 2.70 (95\% CI 2.38-3.06) compared to women and that the better survival in women persisted after adjusting for multiple confounding variables (time period of diagnosis, region, age, Breslow thickness, histological subtype, body site, and nodal and metastatic status) [30].

The role of gender in the survival of melanoma patients was studied in the two most recent studies published by Joosse et al. [31, 32]. In the first study [31], the authors included 2672 melanoma patients from four European Organisation for Research and Treatment of Cancer (EORTC) adjuvant trials. According to their analysis, compared to the male melanoma patients, the female patients appeared to have a highly consistent and independent advantage in OS (adjusted HR 0.70; 95\% CI 0.59-0.83), disease-specific survival (DSS; adjusted HR 0.74; 95\% CI 0.62-0.88), time to lymph node metastasis (adjusted HR $0.70 ; 95 \%$ CI 0.51-0.96), and time to distant metastasis (adjusted HR 0.69; 95\% CI 0.59-0.81). Furthermore, a subgroup analysis showed that the female benefit was consistent across all prognostic subgroups, with the possible exception of head and neck melanomas [31].

In comparison, in our study, we also observed a significant difference according to gender in favor of female patients in median
DFS ( 22 vs. 104 months; $P<0.001$ ) and median OS (20.7 vs. 104 months; $P<0.001)$. Taking the stage at the time of diagnosis also into consideration in a sub-group analysis, we also showed a statistically significant difference in DFS and in OS in favor of females in stages I and II.

In their second study, Joosse et al. considered 2734 patients with stage III melanoma and 1306 patients with stage IV melanoma from five EORTC randomized controlled trials [32]. The results showed that compared to the male patients with stage III melanoma, female patients with stage III melanoma had a better 5 -year DSS rate (51.5 vs. $43.3 \%$ ), an adjusted HR for DSS of 0.85 (95\% CI 0.76-0.95), and an adjusted HR for relapse-free survival of 0.86 (95\% CI 0.77-0.95). The comparison of women and men with stage IV melanoma revealed that the female patients again exhibited an advantage in DSS (2-year survival rate, 14.1 [male] vs. $19.0 \%$ [female]; adjusted HR 0.81; 95\% CI 0.72-0.92) and progression-free survival (adjusted HR 0.79; 95\% CI 0.70-0.88). This female advantage was consistent across pre- and postmenopausal age categories and across different prognostic subgroups. However, the women advantage did appears to become smaller in patients with higher metastatic tumor load [32].

In our study, men and women with stage III and IV melanoma did not show significant sexbased differences in terms of DFS/PFS and OS, possibly due to the small sample of patients with advanced melanoma that were enrolled in the study.

The smaller survival benefit in women in the most advanced stages of melanoma has also been shown in a population-based cohort study performed by Joosse et al., using data on 11,774 melanoma cases extracted from the Munich Cancer Registry (Germany) [33]. In this study, women were found to be at a lower risk of progression (HR 0.68; 95\% CI 0.62-0.75) than men, including a lower risk of lymph node metastasis (HR 0.58; 95\% CI 0.51-0.65) and visceral metastases (HR 0.56; 95\% CI 0.49-0.65). They retained a significant survival advantage over men after the first progression (HR 0.81; 95\% CI 0.71-0.92) and lymph node metastasis (HR 0.80; 95\% CI 0.66-0.96), but this difference became less significant (HR 0.88; 
Table 2 Clinical-pathological features of all melanoma patients enrolled in the study by sex

\begin{tabular}{|c|c|c|c|}
\hline \multirow[t]{2}{*}{ Features } & \multicolumn{3}{|c|}{ Clinical-pathological features of 1023 melanoma patients } \\
\hline & Male patients $(n=487)$ & Female patients $(n=536)$ & $P^{\mathbf{a}}$ \\
\hline \multicolumn{4}{|c|}{ TMN stage (2009 AJCC melanoma staging system) } \\
\hline Stage 0 & $72(14.8 \%)$ & $109(20.3 \%)$ & 0.025 \\
\hline Stage I & $243(49.9 \%)$ & $306(57.2 \%)$ & 0.025 \\
\hline Stage II & $103(21.1 \%)$ & $80(15 \%)$ & 0.012 \\
\hline Stage III & $23(4.7 \%)$ & $17(3.2 \%)$ & 0.264 \\
\hline Stage IV & $14(2.9 \%)$ & $9(1.7 \%)$ & 0.281 \\
\hline Unknown/not applicable & $32(6.6 \%)$ & $15(2.8 \%)$ & - \\
\hline \multicolumn{4}{|l|}{ Pathological TNM stage (pT) } \\
\hline $\mathrm{p}$ Tis & $72(14.8 \%)$ & $107(20.0 \%)$ & 0.036 \\
\hline pT1 & $188(38.6 \%)$ & $241(45.0 \%)$ & 0.046 \\
\hline $\mathrm{pT} 2$ & $78(16.0 \%)$ & $88(16.4 \%)$ & 0.929 \\
\hline pT3 & $64(13.1 \%)$ & $49(9.1 \%)$ & 0.053 \\
\hline pT4 & $50(10.3 \%)$ & $32(6.0 \%)$ & 0.016 \\
\hline Unknown/not applicable & $35(7.2 \%)$ & $19(3.6 \%)$ & - \\
\hline \multicolumn{4}{|l|}{ Histological ulceration } \\
\hline Present & $80(16.4 \%)$ & $52(9.7 \%)$ & 0.002 \\
\hline Absent & $312(64.1 \%)$ & $363(67.7 \%)$ & 0.243 \\
\hline Unknown/not applicable & $95(19.5 \%)$ & $121(22.6 \%)$ & 0.261 \\
\hline \multicolumn{4}{|c|}{ Tumor-infiltrating lymphocytes } \\
\hline Absent & $297(61.0 \%)$ & $348(64.9 \%)$ & 0.215 \\
\hline Non-brisk & $123(25.3 \%)$ & $134(25.0 \%)$ & 0.982 \\
\hline Brisk & $37(7.6 \%)$ & $37(6.9 \%)$ & 0.758 \\
\hline Unknown/not applicable & $30(6.2 \%)$ & $17(3.2 \%)$ & - \\
\hline
\end{tabular}

Values in table are presented as the number (of patients) with the percentage in parenthesis $P \leq 0.05$ indicates a significant difference

${ }^{a}$ Homogeneity of proportions were obtained using the Pearson chi-squared test with Yates' continuity correction, where necessary

95\% CI 0.76-1.03) after visceral metastasis. Localized melanomas in women were found to have a lower propensity to metastasize, resulting in a better survival when compared with men, even after first disease progression [33].
To the best of our knowledge, our study is the largest single-center study carried out in Italy with the aim to investigate gender differences in melanoma with a subgroup analysis.

Explanations for the observed gender difference in melanoma recurrence and survival can 


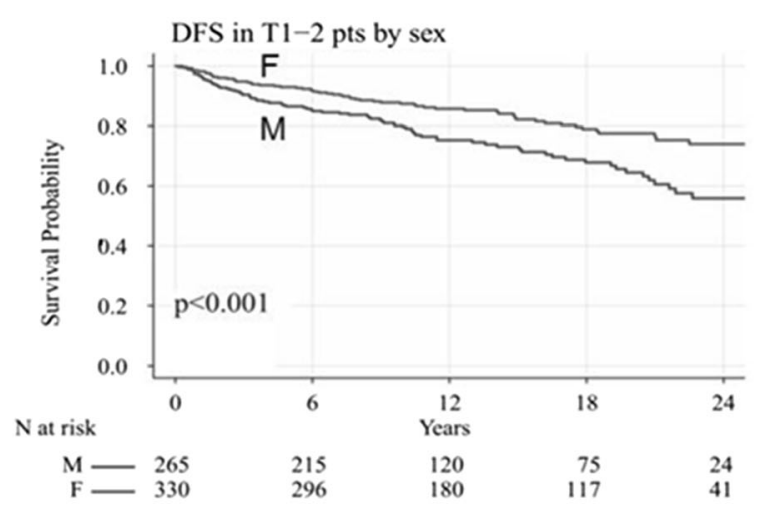

DFS in pts without histological ulceration by sex

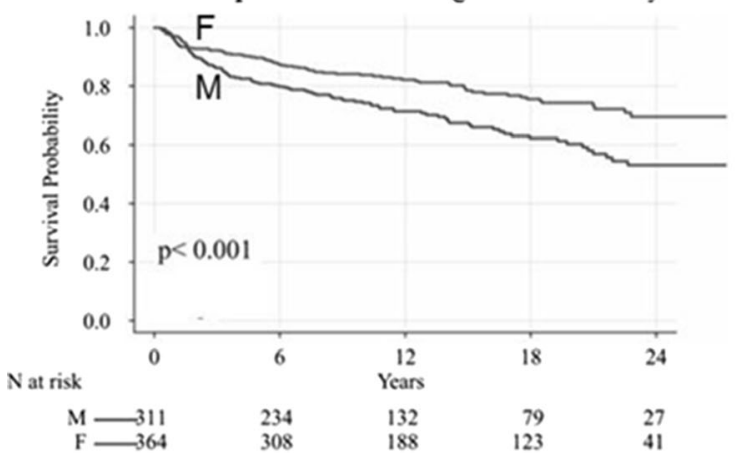

DFS in pts with non-Brisk TILs by sex

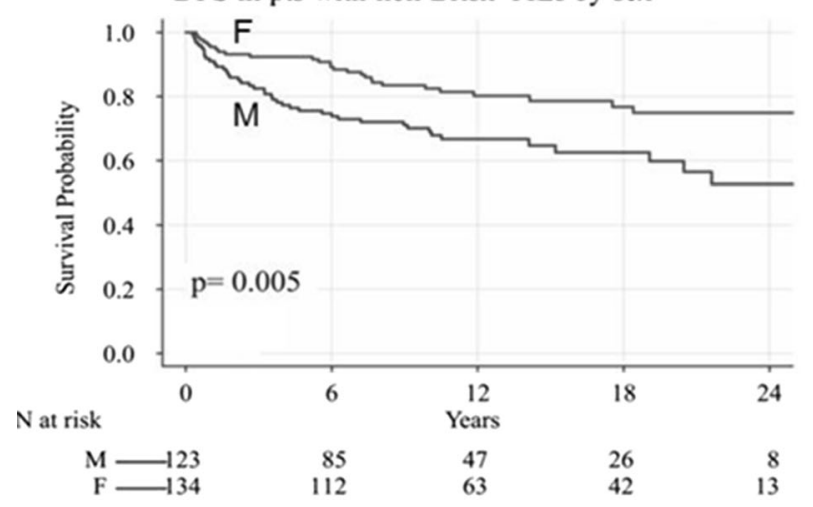

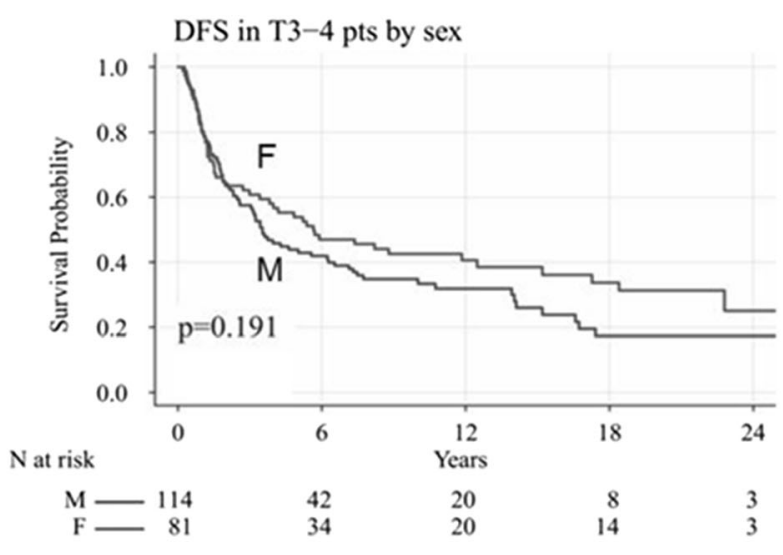
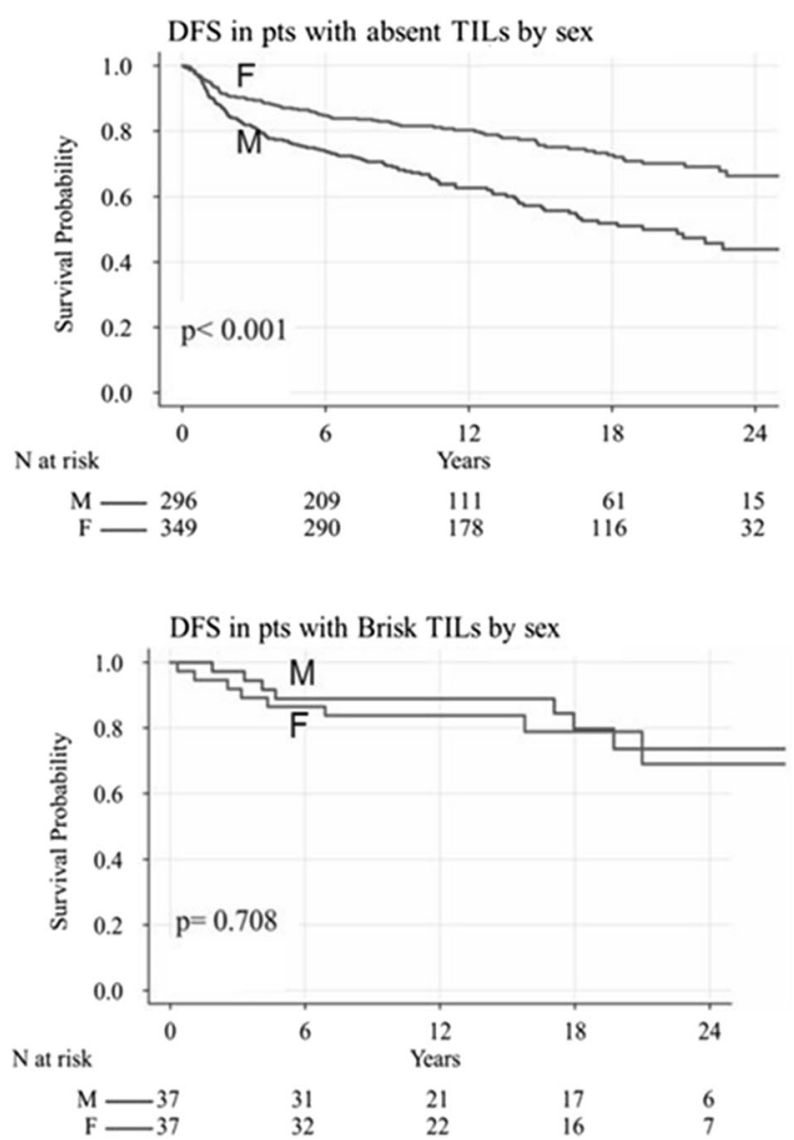

Fig. 1 Disease-free survival $(D F S)$ according to clinical-pathological features by sex. $F$ Female, $M$ male, T1-T4 pts pathological TNM stages $\mathrm{pT} 1-\mathrm{pT}$, TILs tumor-infiltrating lymphocytes

be related to behavioral differences across sex or to biological sex differences affecting melanoma, host-to-tumor relationship, or both. As mentioned earlier in the text, the results of this study provide further evidence that tumor characteristics typically associated with a delayed diagnosis, such as thickness, ulceration, or back localization of primary tumor, even if disadvantageously distributed in men versus women, do not completely explain sex-based survival differences in the early stages of cutaneous melanoma. Furthermore, in cases of 

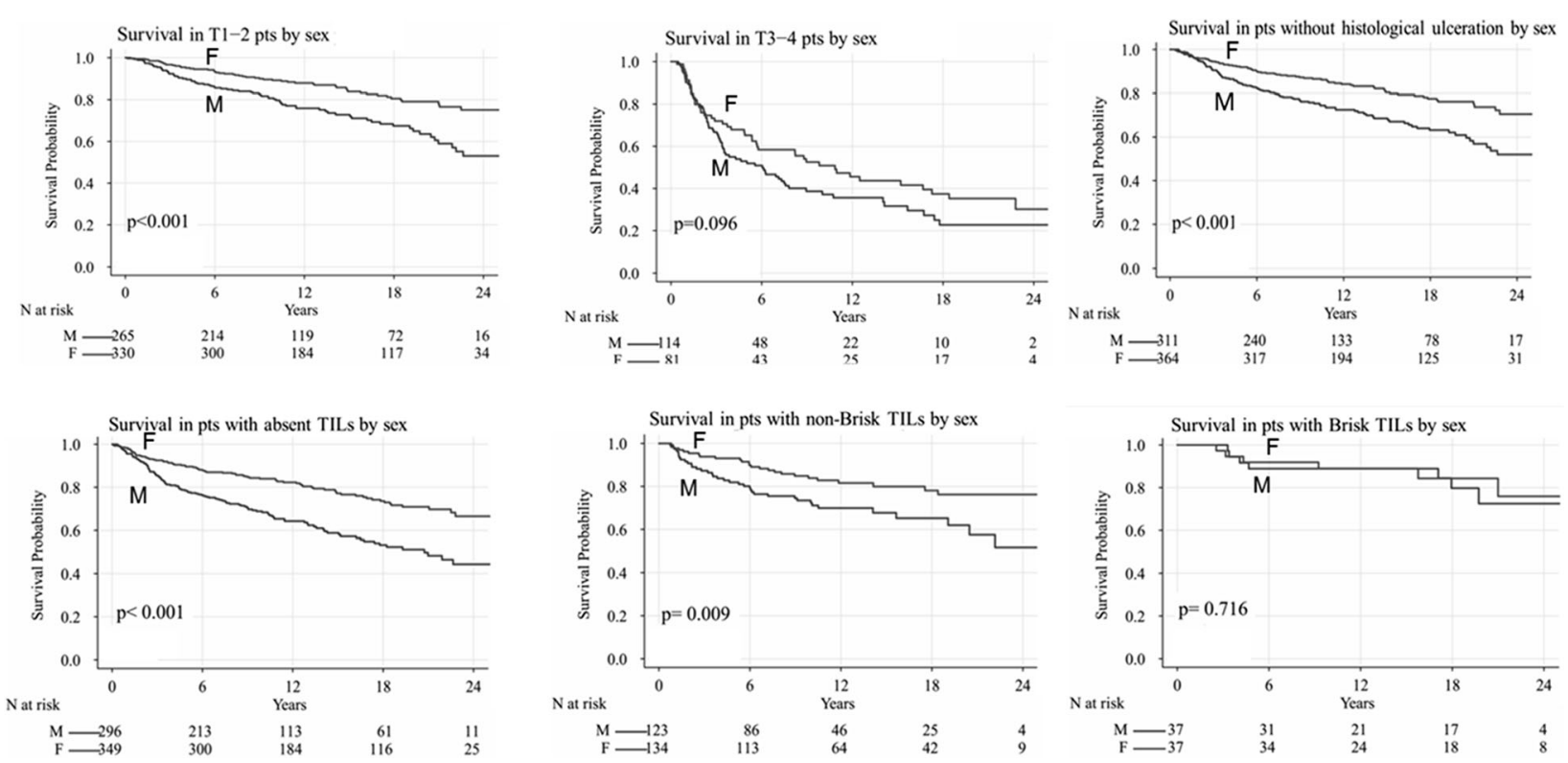

Fig. 2 Overall survival according to clinical-pathological features by sex

advanced melanoma, the effects of skin detection and screening technologies and healthcare consumption are probably even smaller compared with localized melanoma, but a consistent female advantage still exists.

Therefore, it would seem that a biological trait of the tumor or of host-to-tumor relationship that differs across sex profoundly affects melanoma progression and, definitely, the survival of melanoma patients. To our knowledge, a few hypotheses on biological sex differences have been put forward; these include the effect of estrogen receptor expression [34, 35], difference in the capacity of neutralizing oxidative stress [36, 37], different potential protective effect of statin use [38], and possible increased risk of malignant melanoma in overweight and obese male patients [39].

Regarding the potential effect of estrogen levels on the development and progression of cutaneous melanoma, for the endpoints of our study an analysis comparing pre- and postmenopausal age groups was not conducted due to the lack of relevant data. Although the effect of estrogen should not be neglected since the skin has its own hormonal microenvironment and melanomas do express estrogen receptor $\beta$, $[40,41]$, the topic of estrogen regulation of melanoma progression is controversial $[35,42]$.
Various conflicting results have been reported. A majority of studies have reported a persisting women advantage in postmenopausal age groups [30, 33, 43, 44], while other studies observed the women advantage to disappear in postmenopausal age groups [45, 46]. A recent multi-center retrospective analysis revealed that young women with primary melanoma of the trunk may represent a subgroup at higher risk for disease recurrence and metastasis [47].

Other factors seem to be involved in explaining the melanoma gender difference phenomenon. For example, androgen receptors have been observed in melanoma cell lines [48], and sex differences in the capacity to neutralize oxidative stress could be implicated. Other possible explanations for the female advantage that have been less extensively explored in the literature are differences in immune homeostasis $[49,50]$ and vitamin D metabolism $[51,52]$. Last but not least, it has been reported that men and women seem to have different behavior and psychological responses to a stress-related diagnosis of melanoma [53, 54] and that these responses could be another possible-even partial-explanation of gender differences in melanoma progression and survival. 


\section{CONCLUSION}

To summarize, in our study the advantage of female patients with melanoma over their male counterparts was consistent and independent across endpoints. Although this study has a number of limitations, such as the greater number of patients in stage I-II compared to the lower number of patients in a more advanced stage and the absence of correlation of our data with therapy performed by patients, our results strongly suggest that a biological factor underlies the femal advantage. However, further studies aimed at unraveling this underlying cause are necessary. Looking for a biological explanation of gender differences observed in melanoma prognosis could also help to create different screening programs for men and woman, with the aim to better stratify patients and to improve the survival of all melanoma patients.

\section{ACKNOWLEDGEMENTS}

Funding. No funding or sponsorship was received for this study or publication of this article.

Authorship. All named authors meet International Committee of Medical Journal Editors (ICMJE) criteria for authorship for this article, take responsibility for the integrity of the work as a whole and have given their approval for this version to be published. All authors contributed to the editorial, read and approved the final manuscript.

Disclosures. Francesca Morgese, Caterina Sampaolesi, Mariangela Torniai, Alessandro Conti, Nicoletta Ranallo, Alfredo Giacchetti, Stefano Serresi, Azzurra Onofri, Michela Burattini and Giuseppe Ricotti. Rossana Berardi is an Editor-in-Chief for this journal but has no other disclosures to declare.

Compliance with Ethics Guidelines. According to Italian law (resolution March 1, 2012, Gazzetta Ufficiale n.72 of March 26,
2012), ethics approval and informed consent were not required for the present study owing to its retrospective nature, the use of anonymous data and the fact that it was not associated with any change in patients' management. All patients gave their written consent to all the diagnostic and therapeutic procedures.

Data Sharing. The datasets during and/or analyzed during the current study are available from the corresponding author on reasonable request.

Open Access. This article is licensed under a Creative Commons Attribution-NonCommercial 4.0 International License, which permits any non-commercial use, sharing, adaptation, distribution and reproduction in any medium or format, as long as you give appropriate credit to the original author(s) and the source, provide a link to the Creative Commons licence, and indicate if changes were made. The images or other third party material in this article are included in the article's Creative Commons licence, unless indicated otherwise in a credit line to the material. If material is not included in the article's Creative Commons licence and your intended use is not permitted by statutory regulation or exceeds the permitted use, you will need to obtain permission directly from the copyright holder. To view a copy of this licence, visit http://creativecommons.org/licenses/by$\mathrm{nc} / 4.0 /$.

\section{REFERENCES}

1. Bray F, Ferlay J, Soerjomataram I, Siegel RL, Torre LA, Jemal A. Global cancer statistics 2018: GLOBOCAN estimates of incidence and mortality worldwide for 36 cancers in 185 countries. CA Cancer J Clin. 2018;68(6):394-424.

2. Guy GP Jr, Thomas CC, Thompson T, Watson M, Massetti GM, Richardson LC. Vital signs: melanoma incidence and mortality trends and projectionsUnited States, 1982-2030. MMWR Morb Mortal Wkly Rep. 2015;64(21):591-6.

3. National Cancer Institute. Surveillance, Epidemiology, and End Results Program. Previous version: 
SEER cancer statistics review, 1975-2013. 2015. http://seer.cancer.gov/csr/1975_2013/.

4. World Health Organization. Global solar UV index: a practical guide. 2002. http://www.who.int/uv/ publications/en/GlobalUVI.pdf?ua=1. Accessed 24 Oct 2017.

5. American Cancer Society. Cancer facts and figures 2019. Atlanta: American Cancer Society. 2019. https://www.cancer.org/research/cancer-facts-statis tics/all-cancer-facts-figures/cancer-facts-figures-2019. html. Accessed July 2019.

6. Shen W, Sakamoto N, Yang L. Melanoma-specific mortality and competing mortality in patients with non-metastatic malignant melanoma: a population-based analysis. BMC Cancer. 2016;16:413.

7. Rastrelli M, Tropea S, Rossi C, Alaibac M. Melanoma: epidemiology, risk factors, pathogenesis, diagnosis and classification. In Vivo. 2014;28:1005-11.

8. Siegel R, Ma J, Zou Z, Jemal A. Cancer Statistics 2014. CA Cancer J Clin. 2014;64(1):9-29.

9. Ferlay J, Shin HR, Bray F, Forman D, Mathers C, Parkin DM. Cancer incidence and mortality worldwide: GLOBOCAN 2008 v2.0. CancerBase No. 10. Lyon: International Agency for Research. 2010. http://globocan.iarc.fr.

10. Apalla Z, Nashan D, Weller RB, Castellsague X. Skin cancer: epidemiology, disease burden, pathophysiology, diagnosis, and therapeutic approaches. Dermatol Ther. 2017;7[Suppl 1]:5-19.

11. Whiteman DC, Green AC, Olsen CM. The growing burden of invasive melanoma: projections of incidence rates and numbers of new cases in six susceptible populations through 2031. J Investig Dermatol. 2016;136(6):1161-71.

12. AIOM-CCM-AIRTUM I numeri del cancro in Italia. 2018. http://www.registri-tumori.it/cms/.

13. Bianconi F, Crocetti E, Grisci C, Primieri C, Stracci F. What has changed in the epidemiology of skin melanoma in central Italy during the past 20 years? Melanoma Res. 2018. https://doi.org/10.1097/ CMR.0000000000000551.

14. I numeri del Cancro in Italia. Rapporto AIOM-AIRTUM. 2017. http://www.registri-tumori.it/cms/.

15. De Angelis R, Sant M, Coleman MP, et al. Cancer survival in Europe 1999-2007 by country and age: results of EUROCARE-5-a population-based study. Lancet Oncol. 2014;15(1):23-34.

16. Micheli A, Ciampichini R, Oberaigner W, et al. The advantage of women in cancer survival: an analysis of EUROCARE-4 data. Eur J Cancer. 2009;45(6): 1017-27.

17. Rigel DS. Epidemiology of melanoma. Semin Cutan Med Surg. 2010;29(4):204-9.

18. Weyers W. The 'epidemic' of melanoma between under- and overdiagnosis. J Cutan Pathol. 2012;39: 9-16.

19. Mandalà M, Rossi CR, De Giorgi V, et al. Melanoma cutaneo. In: Labianca R, Cascinu S, editors. La medicina oncologica. Milan: Edizioni Edra; 2013:41-75.

20. Howlader N, Noone AM, Krapcho M, et al. Cancer statistics review, 1975-2012. Bethesda: National Cancer Institute. http://seer.cancer.gov/csr/1975_ 2012/, based on November 2014 SEER data submission, posted to the SEER website April 2015.

21. Erturk K, Tas F. Effect of biology on the outcome of female melanoma patients. Mol Clin Oncol. 2017;7(6):1093-100.

22. Balch CM, Gershenwald JE, Soong SJ, et al. Final version of 2009 AJCC melanoma staging and classification. J Clin Oncol. 2009;27:6199-206.

23. Haanen JB, Baars A, Gomez R, et al. Melanomaspecific tumor-infiltrating lymphocytes but not circulating melanoma-specific T cells may predict survival in resected advanced-stage melanoma patients. Cancer Immunol Immunother. 2006;55:451-8.

24. Azimi F, Scolyer RA, Rumcheva P, et al. Tumor-infiltrating lymphocyte grade is an independent predictor of sentinel lymph node status and survival in patients with cutaneous melanoma. J Clin Oncol. 2012;30:2678-83.

25. Khosrotehrani K, Dasgupta P, Byrom L, Youlden DR, Baade PD, Green AC. Melanoma survival is superior in females across all tumour stages but is influenced by age. Arch Dermatol Res. 2015;307(8):731-40.

26. Siegel R, Ward E, Brawley O, Jemal A. Cancer statistics, 2011: the impact of eliminating socioeconomic and racial disparities on premature cancer deaths. CA Cancer J Clin. 2011;61:212-36.

27. Collins KK, Fields RC, Baptiste D, Liu Y, Moley J, Jeffe DB. Racial differences in survival after surgical treatment for melanoma. Ann Surg Oncol. 2011;18: 2925-36.

28. Martinez SR, Tseng WH, Young SE. Outcomes for lymph node-positive cutaneous melanoma over two decades. World J Surg. 2011;35:1567-72.

29. Farahi JM, Fazzari M, Braunberger T, et al. Gender differences in melanoma prognostic factors. Dermatol Online J. 2018; 24(4). 
30. de Vries E, Nijsten TE, Visser O, et al. Superior survival of females among 10.538 Dutch melanoma patients is independent of Breslow thickness, histologic type and tumor site. Ann Oncol. 2008;19:583-9.

31. Joosse A, Collette S, Suciu S, et al. Superior outcome of women with stage I/II cutaneous melanoma: pooled analysis of four European organisation for research and treatment of cancer phase III trials. J Clin Oncol. 2012;30(18):2240-7.

32. Joosse A, Collette S, Suciu S, et al. Sex is an independent prognostic indicator for survival and relapse/progression free-survival in metastasized stage III to stage IV melanoma: a pooled analysis of five European organization for research and treatment on cancer randomized controlled trials. J Clin Oncol. 2013;31(18):2337-46.

33. Joosse A, de Vries E, Eckel R, et al. Gender differences in melanoma survival: female patients have a decreased risk of metastasis. J Invest Dermatol. 2011;131:719-26.

34. Spyropoulos C, Melachrinou M, Vasilakos P, Tzorakoleftherakis E. Expression of estrogen receptors in melanoma and sentinel lymph nodes; a "female" clinical entity or a possible treatment modality? Eur J Gynaecol Oncol. 2015;36(2):123-30.

35. Janik Marcelina E, Bełkot Klaudyna, Przybyło Małgorzata. Is oestrogen an important player in melanoma progression? Contemp Oncol (Pozn). $2014 ; 18(5): 302-6$.

36. Bechmann N, Kniess T, Pietzsch J. Nitric oxide-releasing selective estrogen receptor modulators: a bifunctional approach to improve the therapeutic index. J Med Chem. 2019;62(14):6525-39.

37. Joosse A, de Vries E, van Eijck CH, Eggermont AM, Nijsten T, Coebergh JW. Reactive oxygen species and melanoma: an explanation for gender differences in survival? Pigment Cell Melanoma Res. 2010;23:352-64.

38. Sarrabayrouse G, Pich C, Teiti I, Tilkin-Mariame AF. Regulatory properties of statins and rho GTPases prenylation inhibitors to stimulate melanoma immunogenicity and promote anti-melanoma immune response. Int J Cancer. 2017;140(4): 747-55.

39. Clement E, Lazar I, Muller C, Nieto L. Obesity and melanoma: could fat be fueling malignancy? Pigment Cell Melanoma Res. 2017;30(3):294-306.

40. Marzagalli M, Montagnani Marelli M, Casati L, Fontana F, Moretti RM, Limonta P. Estrogen receptor $\beta$ in melanoma: from molecular insights to potential clinical utility. Front Endocrinol (Lausanne). 2016;7:140.
41. Gori A, Savarese I, D'Errico A, et al. Estrogen receptor (ER) $\beta$ expression and worse outcome from melanoma in pregnant and perimenopausal women. J Am Acad Dermatol. 2016;75(3):e117.

42. Gupta A, Driscoll MS. Do hormones influence melanoma? Facts and controversies. Clin Dermatol. 2010;28:287-92.

43. Caruntu C, Mirica A, Roşca AE, et al. The role of estrogens and estrogen receptors in melanoma development and progression. Acta Endocrinol (Buchar). 2016;12(2):234-41.

44. Donley GM, Liu WT, Pfeiffer RM, et al. Reproductive factors, exogenous hormone use and incidence of melanoma among women in the United States. Br J Cancer. 2019;120(7):754-60.

45. Kemeny MM, Busch E, Stewart AK, Menck HR. Superior survival of young women with malignant melanoma. Am J Surg. 1999;175:437-44.

46. Lasithiotakis K, Leiter U, Meier F, et al. Age and gender are significant independent predictors of survival in primary cutaneous melanoma. Cancer. 2008;112:1795-804.

47. Burton AL, Egger ME, Quillo AR, et al. Prognostic factors in young women with cutaneous melanoma. Am J Surg. 2014;207(1):102-8.

48. Mitkov M, Joseph R, Copland J 3rd. Steroid hormone influence on melanomagenesis. Mol Cell Endocrinol. 2015;417:94-102.

49. Bouman A, Heineman MJ, Faas MM. Sex hormones and the immune response in humans. Hum Reprod Update. 2005;11:411-23.

50. Chen W, Mempel M, Traidl-Hofmann C, Al Khusaei S, Ring J. Gender aspects in skin disease. J Eur Acad Dermatol Venereol. 2010;24:1378-85.

51. Slominski AT, Brożyna AA, Zmijewski MA, et al. Vitamin D signaling and melanoma: role of vitamin $\mathrm{D}$ and its receptors in melanoma progression and management. Lab Invest. 2017;97(6):706-24.

52. Vasilovici AF, Grigore LE, Ungureanu L, et al. Vitamin D receptor polymorphisms and melanoma. Oncol Lett. 2019;17(5):4162-9.

53. Hajdarevic S, Schmitt-Egenolf M, Sundbom E, Isaksson U, Hörnsten A. Coping styles in decisionmaking among men and women diagnosed with malignant melanoma. J Health Psychol. 2013;18(11):1445-55.

54. Hamama-Raz Y. Does psychological adjustment of melanoma survivors differs between genders? Psychooncology. 2012;21(3):255-63. 